\title{
SARS-CoV-2 RBD219-N1C1: A Yeast-Expressed SARS-CoV-2 \\ Recombinant Receptor-Binding Domain Candidate Vaccine Stimulates Virus Neutralizing Antibodies and T-cell Immunity in Mice.
}

Jeroen Pollet ${ }^{1,2}$, Wen-Hsiang Chen ${ }^{1,2}$, Leroy Versteeg ${ }^{1}$, Brian Keegan ${ }^{1}$, Bin Zhan ${ }^{1,2}$, Junfei Wei ${ }^{1}$, Zhuyun Liu ${ }^{1}$, Jungsoon Lee ${ }^{1}$, Rahki Kundu ${ }^{1}$, Rakesh Adhikari ${ }^{1}$, Cristina Poveda ${ }^{1}$, Maria Jose Villar ${ }^{1}$, Ana Carolina de Araujo Leao $^{1}$, Joanne Altieri Rivera ${ }^{1}$, Zoha Momin ${ }^{3}$, Portia M. Gillespie ${ }^{1}$, Jason T. Kimata $^{3}$, Ulrich Strych ${ }^{1,2}$, Peter J. Hotez ${ }^{1,2,4,5}$, Maria Elena Bottazzi ${ }^{1,2,4^{*}}$

${ }^{1}$ Texas Children's Hospital Center for Vaccine Development, Houston, TX, USA

${ }^{2}$ Departments of Pediatrics and Molecular Virology \& Microbiology, National School of Tropical Medicine, Baylor College of Medicine, Houston, TX, USA

${ }^{3}$ Department of Molecular Virology and Microbiology, Baylor College of Medicine,

Houston, TX, USA

${ }^{4}$ Department of Biology, Baylor University, Waco, TX, USA

${ }^{5}$ James A. Baker III Institute for Public Policy, Rice University, Houston, TX, USA

Corresponding Author:

bottazzi@bcm.edu 


\title{
SARS-CoV-2 RBD219-N1C1: A Yeast-Expressed SARS-CoV-2 Recombinant Receptor-Binding Domain Candidate Vaccine Stimulates Virus Neutralizing Antibodies and T-cell Immunity in Mice
}

\author{
Abstract \\ There is an urgent need for an accessible and low-cost COVID-19 vaccine \\ suitable for low- and middle-income countries. Here we report on the \\ development of a SARS-CoV-2 receptor-binding domain (RBD) protein, \\ expressed at high levels in yeast (Pichia pastoris), as a suitable vaccine candidate \\ against COVID-19. After introducing two modifications into the wild-type RBD \\ gene to reduce yeast-derived hyperglycosylation and improve stability during \\ protein expression, we show that the recombinant protein, RBD219-N1C1, is \\ equivalent to the wild-type RBD recombinant protein (RBD219-WT) in an in \\ vitro ACE-2 binding assay. Immunogenicity studies of RBD219-N1C1 and \\ RBD219-WT proteins formulated with Alhydrogel ${ }^{\circledR}$ were conducted in mice, \\ and, after two doses, both the RBD219-WT and RBD219-N1C1 vaccines induced \\ high levels of binding IgG antibodies. Using a SARS-CoV-2 pseudovirus, we \\ further showed that sera obtained after a two-dose immunization schedule of the \\ vaccines were sufficient to elicit strong neutralizing antibody titers in the 1:1,000 \\ to 1:10,000 range, for both antigens tested. The vaccines induced IFN- $\gamma$, IL-6, \\ and IL-10 secretion, among other cytokines. Overall, these data suggest that the \\ RBD219-N1C1 recombinant protein, produced in yeast, is suitable for further \\ evaluation as a human COVID-19 vaccine, in particular, in an Alhydrogel ${ }^{\circledR}$ \\ containing formulation and possibly in combination with other \\ immunostimulants.
}

Keywords: COVID-19, RBD, alum, pseudovirus, coronavirus, ACE-2 


\section{Introduction}

The number of coronavirus disease 19 (COVID-19) cases globally is readily over the 100-million-person mark, with over 2 million deaths up to early February 2021. In response to the pandemic, several vaccines have been shown to be effective and have been either approved or authorized for emergency use in different countries ${ }^{1}$. There are many ways to categorize the more than 100 potential COVID-19 vaccine candidates ${ }^{2}$, but one approach is to divide them as those employing novel, not hitherto licensed technologies for production, versus those employing traditional vaccine production approaches with precedence in licensed vaccines ${ }^{3}$. The Operation Warp Speed (OWS) initiative in the United States ${ }^{4}$ and similar efforts in other parts of the world ${ }^{5}$ initially focussed on approaches employing new platforms, including several messenger RNA (mRNA)-based vaccines as well as non-replicating adenovirus vector vaccines ${ }^{4}$. Among the more established or traditional approaches, whole-inactivated virus vaccines on aluminum oxy-hydroxide have been developed in China ${ }^{6}$, as have several recombinant protein vaccine candidates ${ }^{7-10}$. Each of these approaches offers both distinct advantages and disadvantages in terms of production, scale-up, potential efficacy, and safety, as well as delivery.

We have previously reported on recombinant protein-based coronavirus vaccine candidates, formulated with Alhydrogel ${ }^{\circledR}$ to prevent Severe Acute Respiratory Syndrome (SARS) $)^{11-13}$ and Middle East Respiratory Syndrome (MERS) ${ }^{14}$. In both cases, the Receptor-Binding Domain (RBD) of the SARS or MERS spike proteins was used as the target vaccine antigen. In a mouse model, the SARS-CoV RBD219N1/Alhydrogel ${ }^{\circledR}$ vaccine induced high titers of virus-neutralizing antibodies and protective immunity against a mouse-adapted SARS-CoV virus challenge. It was also 
found to minimize or prevent eosinophilic immune enhancement when compared with the full spike protein ${ }^{11}$.

The RBD of SARS-CoV-2 has likewise attracted interest from several groups now entering clinical trials ${ }^{8,15-19}$. Our original approach was to apply the lessons learned from SARS-CoV and accelerate COVID-19 vaccine development efforts using microbial fermentation in the yeast Pichia pastoris $^{20}$. We selected the SARS-CoV-2 RBD219-WT sequence, residues 331-549, through alignment with the SARS-CoV RBD sequence, and later developed a genetically engineered version (RBD219-N1C1) to improve antigen stability ${ }^{21}$.

Yeast expression technology is widely available and used to produce, for example, the VLP antigen of the licensed HPV vaccine as well as recombinant hepatitis B vaccines in several middle-income countries (LMICs) ${ }^{22}$, including Bangladesh, Brazil, Cuba, India, and Indonesia over the last 35 years.

As COVID-19 spreads across the globe, especially among urban populations living in extreme poverty ${ }^{23}$, there will be greater urgency to produce safe, effective, highly scalable, and affordable COVID-19 vaccines locally or regionally. Therefore, the development of a yeast-expressed recombinant protein-based COVID-19 vaccine allows developing it for global health and populations vulnerable to poverty-related diseases ${ }^{22}$.

Here, we present the first preclinical data of a COVID-19 recombinant proteinbased vaccine candidate, SARS-CoV2 RBD219-N1C1, formulated with Alhydrogel ${ }^{\circledR}$. We demonstrate that modifications made to the SARS-CoV2 RBD gene to improve production and stability preserve the protein antigen functionality and its immunogenicity after Alhydrogel ${ }^{\circledR}$ adsorption. 


\section{Materials and Methods}

\subsection{Cloning and expression of the genes encoding RBD219-WT and RBD219- N1C1:}

The RBD219-WT recombinant subunit protein contains amino acid residues 331-549 of the SARS-CoV-2 spike protein (GenBank No.: QHD43416.1). It contains a hexahistidine tag at its C-terminus. In the tag-free RBD219-N1C1 antigen candidate, the N331 glycosylation site has been removed, and C538 has been mutated to an alanine residue to prevent aggregation due to intermolecular disulfide bonding. The DNAs for both antigen candidates were individually synthesized with their codon use optimized for translation in Pichia pastoris and ligated into pPICZ $\alpha$ A using the EcoRI and XbaI restriction sites (GenScript). The recombinant plasmids were electroporated into $P$. pastoris X33 following the EasySelect ${ }^{\mathrm{TM}}$ Pichia Expression Kit Manual (Invitrogen). Transformants were selected on YPD plates containing different concentrations of Zeocin $(100-2000 \mu \mathrm{g} / \mathrm{mL})$ and incubated at $30^{\circ} \mathrm{C}$ for 72 hours. Individual colonies were screened for expression under induction with methanol (0.5-2\%) at the $10 \mathrm{~mL}$ culture level (BMMY medium) as described ${ }^{12,22}$. The expression level of select colonies was identified by SDS-PAGE and immunoblotting using anti-SARS-CoV-2 antibodies (antiSARS-CoV-2 spike rabbit monoclonal antibody, Sino Biological, Cat \# 40150-R007), and research seed stocks of the highest expressing clones were frozen at $-80{ }^{\circ} \mathrm{C}$.

RBD219-WT and RBD219-N1C1 were expressed at the 5 L scale using a Celligen 310 benchtop fermentation system (Eppendorf). For the RBD-WT, $2.5 \mathrm{~L}$ of basal salt medium were inoculated with a seed culture to an initial $\mathrm{OD}_{600}$ of 0.05 and grown at $30{ }^{\circ} \mathrm{C}$, pH 5.0 with $30 \%$ dissolved oxygen until glycerol depletion. During the first hour of methanol induction, the temperature was reduced from $30{ }^{\circ} \mathrm{C}$ to $26{ }^{\circ} \mathrm{C}$ and the $\mathrm{pH}$ was increased from 5.0 to 6.0 . After approximately 70 hours of induction 
(methanol feed at 1-11 mL/L/hr), the culture was harvested from the fermenter, and cells were removed by centrifugation for $30 \mathrm{~min}$ at $12,227 \mathrm{x}$ g at $4{ }^{\circ} \mathrm{C}$. For RBD219$\mathrm{N} 1 \mathrm{C} 1$, the fermentation process was slightly different in that low salt medium was used, the induction temperature was set to $25^{\circ} \mathrm{C}$ and the $\mathrm{pH}$ to 6.5 and, the methanol feed rate was between $1-15 \mathrm{ml} / \mathrm{L} / \mathrm{hr}$. The fermentation supernatant (FS) was filtered $(0.45 \mu \mathrm{m}$ PES filter) and stored at $-80{ }^{\circ} \mathrm{C}$ before purification.

A hexahistidine-tagged SARS-CoV-2 RBD219-WT was purified from fermentation supernatant (FS) by immobilized metal affinity chromatography followed by size exclusion chromatography (SEC). The FS was concentrated, and buffer exchanged to buffer A (20 mM Tris- $\mathrm{HCl} \mathrm{pH} 7.5$ and $0.5 \mathrm{M} \mathrm{NaCl})$ using a Pellicon 2 cassette with a $10 \mathrm{kDa}$ MWCO membrane (MilliporeSigma) before being applied to a Ni-Sepharose column (Cytiva). The column was washed with buffer A plus $30 \mathrm{mM}$ imidazole and elution was undertaken in buffer A containing $250 \mathrm{mM}$ imidazole. The RBD219-WT protein was further purified using a Superdex 75 prep grade column (Cytiva) pre-equilibrated in buffer B (20 mM Tris- $\mathrm{HCl}$ pH 7.5 and $150 \mathrm{mM} \mathrm{NaCl})$ after concentrating eluates from the Ni column using an Amicon Ultra-15 concentrator with a 10 kDa MWCO membrane (MilliporeSigma). Monomeric RBD219-WT was pooled, aseptically filtered using a $0.22 \mu \mathrm{m}$ filter, and stored at $-80{ }^{\circ} \mathrm{C}$.

For the purification of the tag-less RBD219-N1C1, ammonium sulfate salt was added to the FS to a final concentration of $1 \mathrm{M}(\mathrm{w} / \mathrm{v})$ before the sample was applied to a Butyl Sepharose HP column (Cytiva). The column was washed with buffer C (30 mM Tris- $\mathrm{HCl} \mathrm{pH}$ 8.0) with $1 \mathrm{M}$ ammonium sulfate and protein was eluted with buffer $\mathrm{C}$ containing 0.4 M ammonium sulfate. A polishing step using a Superdex 75 prep grade column (Cytiva) pre-equilibrated in buffer B followed. 


\subsection{SDS-PAGE}

To evaluate the size of RBD219-WT and RBD219-N1C1, $2 \mu \mathrm{g}$ of these two proteins were loaded onto a 4-20\% tris-glycine gel under non-reduced and reduced conditions. These two proteins were also treated with PNGase-F (NEB) under the reduced condition to remove N-glycans and loaded on the gel to assess the impact of the glycans on the protein size. Gels were stained using Coomassie Blue and analyzed using a BioRad G900 densitometer with Image Lab software.

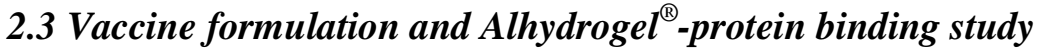

SARS-CoV-2 RBD219-N1C1 was diluted in 20 mM Tris, $150 \mathrm{mM} \mathrm{NaCl,} \mathrm{pH} 7.5$ (TBS buffer) before mixing with Alhydrogel ${ }^{\circledR}$ (aluminum oxy-hydroxide; Catalog \# 250843261 EP, Brenntag). To calculate the Langmuir binding isotherm of RBD219-N1C1 to Alhydrogel ${ }^{\circledR}$, RBD219-N1C1 and Alhydrogel ${ }^{\circledR}$ were mixed at different ratios (from 1:2 to 1:20). The RBD219-N1C1/Alhydrogel ${ }^{\circledR}$ mixture was stored for one hour at RT, to reach an equilibrium state. The Alhydrogel ${ }^{\circledR}$ formulations were centrifuged at 13,000 $\mathrm{x} \mathrm{g}$ for $5 \mathrm{~min}$, and the supernatant was removed. The protein in the supernatant fraction and the pellet fraction were quantified using a micro-BCA assay (ThermoFisher).

\subsection{ACE-2 binding assay}

For the ACE-2 binding study, the Alhydrogel ${ }^{\circledR}$-RBD vaccine formulations were blocked overnight with $0.1 \%$ BSA. After hACE-2-Fc (LakePharma) was added, the samples were incubated for 2 hours at RT. After incubation, the Alhydrogel ${ }^{\circledR}$ was spun down at $13,000 \times \mathrm{g}$ for $5 \mathrm{~min}$. The hACE-2-Fc which did not bind to the RBD on the Alhydroge ${ }^{\circledR}$ remained in the supernatant. The hACE-2-Fc content in the supernatant was quantified by ELISA using 96-Well MaxiSorp Immuno plates (ThermoFisher) coated overnight with $200 \mathrm{ng} /$ well of RBD219-WT protein. After blocking with $0.1 \%$ 
BSA, $100 \mu \mathrm{L}$ supernatant samples were added to each well. Plates were washed 4 times with an automated plate washer using PBS with Tween (PBST). A secondary antibody against human Fc was used to detect hACE-2-Fc bound the proteins on the plate. Plates were washed 5 times with an automated plate washer using PBST before $100 \mu \mathrm{L}$ TMB solution were added. The enzymatic reaction was stopped with $\mathrm{HCl}$ and absorption readings were made at $450 \mathrm{~nm}$. The final concentration of the hACE- 2 bound on the Alhydrogel $^{\circledR}$ was determined as $\left[\mathrm{hACE}-2-\mathrm{Fc}\right.$ on Alhydrogel $\left.{ }^{\circledR}\right]=[$ Total hACE-2-Fc $]-$ [hACE-2-Fc in supernatant].

\subsection{Immunogenicity testing}

To examine RBD-specific antibodies in mouse sera, indirect ELISAs were conducted. 96-well NUNC ELISA plates were coated with $2 \mu \mathrm{g} / \mathrm{mL}$ RBD219-WT in $100 \mu \mathrm{L} 1 \mathrm{x}$ coating buffer per well and incubated overnight at $4{ }^{\circ} \mathrm{C}$. The next day the coating buffer was discarded, and plates were blocked with $200 \mu \mathrm{L} /$ well $0.1 \%$ BSA in PBST for 2 hours at room temperature. Mouse serum samples were diluted from 1:200 to 1:

437,400 in $0.1 \%$ BSA in PBST. Blocked ELISA plates were washed once with $300 \mu \mathrm{L}$ PBST using a Biotek 405TS plate washer and diluted mouse serum samples were added to the plate in duplicate, $100 \mu \mathrm{L} /$ well. As negative controls, pooled naïve mouse serum (1:200 diluted) and blanks (0.1\% BSA PBST) were added as well. Plates were incubated for 2 hours at room temperature, before being were washed four times with PBST. Subsequently, 1:6,000 diluted goat anti-mouse IgG HRP antibody (100 $\mu \mathrm{L} /$ well) was added in $0.1 \%$ BSA in PBST. Plates were incubated for 1 hour at room temperature, before washing five times with PBST, followed by the addition of 100 $\mu \mathrm{L} /$ well TMB substrate. Plates were incubated for $15 \mathrm{~min}$ at room temperature while protected from light. After incubation, the reaction was stopped by adding $100 \mu \mathrm{L} /$ well 
$1 \mathrm{M} \mathrm{HCl}$. The absorbance at a wavelength of $450 \mathrm{~nm}$ was measured using a BioTek Epoch 2 spectrophotometer. Duplicate values of raw data from the $\mathrm{OD}_{450}$ were averaged. The titer cutoff value was calculated using the following formula: Titer cutoff $=3 \mathrm{x}$ average of negative control $+3 \mathrm{x}$ standard deviation of the negative control. For each sample, the titer was determined as the lowest dilution of each mouse sample with an average $\mathrm{OD}_{450}$ value above the titer cutoff. When a serum sample did not show any signal at all and a titer could not be calculated, an arbitrary baseline titer value of 67 was assigned to that sample (baseline).

\subsection{Pseudovirus assay:}

Pseudovirus was prepared in HEK-293T cells by previously reported methods with modifications ${ }^{24}$. Cells were transfected with $2.5 \mu \mathrm{g}$ of the plasmid encoding the SARSCoV-2 spike protein (p278-1 ${ }^{25}$ ) and $3.7 \mu \mathrm{g}$ of luciferase-encoding reporter plasmid (pNL4-3.lucR-E ${ }^{26}$ ) and Gag/Pol-encoding packaging construct $\left(\mathrm{p} \Delta 8.9^{27}\right)$. Pseudovirus containing supernatant was recovered after $48 \square \mathrm{h}$ and passed through a $0.45 \mu \mathrm{M}$ filter before use.

For each serum sample, $30 \mu \mathrm{L}$ pseudovirus were incubated with serial dilutions of heat-inactivated serum (eight dilutions in a 4-fold stepwise manner) for $1 \mathrm{~h}$ at $37^{\circ} \mathrm{C}$. Next, $100 \mu \mathrm{L}$ of these sera-pseudovirus mixtures were added to 293T-hACE2 cells in 96-well poly-D-lysine coated culture plates. Following $48 \mathrm{~h}$ incubation in a 5\% CO2 environment at $37^{\circ} \mathrm{C}$, the cells were lysed with $100 \mu \mathrm{L}$ Promega Glo Lysis buffer, 15 min RT. Finally, $20 \mu \mathrm{L}$ lysate was added to $100 \mu \mathrm{L}$ Luc substrate (Promega Luciferase Assay System). The amount of luciferase was quantified by luminescence (relative luminescence units (RLU)), using a Promega GloMax luminometer (Steady-Glo program). The percent virus inhibition was calculated as (1- RLU of sample/ RLU of negative control) x 100. Serum from vaccinated mice was also characterized by the 
IC50-value, defined as the serum dilution at which the virus infection was reduced to $50 \%$ compared with the negative control (virus $\square+\square$ cells). When a serum sample did not neutralize $50 \%$ of the virus when added at a 1:10 dilution, the IC50 titer could not be calculated and an arbitrary baseline titer value of 10 was assigned to that sample (baseline). As a control, human convalescent sera for SARS-CoV-2 (NIBSC 20/130) were used (National Institute for Biological Standards and Control).

\subsection{Cytokine analysis}

\subsubsection{Preparation of splenocytes for restimulation}

Single-cell suspensions from mouse splenocytes were prepared using a cell dissociator (GentleMACS Octo Dissociator, Miltenyi Biotec) based on a previously optimized protocol $^{28}$. The concentration and the viability of the splenocyte suspensions were measured after mixing with AOPI dye and counted using the Nexcelom Cellometer Auto 2000.

For the re-stimulation assays, splenocyte suspensions were diluted to $8 \times 10^{6}$ live cells $/ \mathrm{mL}$ in a $2-\mathrm{mL}$ deep-well dilution plate and $125 \mu \mathrm{L}$ of each sample was seeded in two 96-well tissue culture treated culture plates. Splenocytes were re-stimulated with 10 $\mu \mathrm{g} / \mathrm{mL}$ RBD219-WT, $20 \mathrm{ng} / \mathrm{mL}$ PMA $+1 \mu \mathrm{g} / \mathrm{mL}$ Ionomycin or just media (unstimulated). For the flow cytometry plate, the PMA/I was not added until the next day. $125 \mu \mathrm{L}$ ( $2 \mathrm{x}$ concentration) of each stimulant was mixed with the $125 \mu \mathrm{L}$ splenocytes suspension in the designated wells. After all the wells were prepared, the plates were incubated at $37{ }^{\circ} \mathrm{C} 5 \% \mathrm{CO}_{2}$. One plate was used for the cytokine release assay, while the other plate was used for flow cytometry. For flow cytometry, another plate was prepared with splenocytes, which would be later used as fluorescence minus one - controls (FMOs). 


\subsubsection{In vitro cytokine release assay}

After 48 hours in the incubator, splenocytes were briefly mixed by pipetting. Then plates were centrifuged for $5 \mathrm{~min}$ at $400 \mathrm{x} \mathrm{g}$ at RT. Without disturbing the pellet $50 \mu \mathrm{L}$ supernatant was transferred to two skirted PCR plates and frozen at $-20{ }^{\circ} \mathrm{C}$ until use.

For the in vitro cytokine release assay, splenocytes were seeded in a 96-well culture plate at $1 \times 10^{6}$ live cells in $250 \mu \mathrm{L}$ cRPMI. Splenocytes were then (re-)stimulated with either $10 \mu \mathrm{g} / \mathrm{mL}$ RBD219-WT protein, $10 \mu \mathrm{g} / \mathrm{mL}$ RBD219-N1C1 protein, PMA/I (positive control), or nothing (negative control) for 48 hours at $37{ }^{\circ} \mathrm{C} 5 \% \mathrm{CO}_{2}$. After incubation, 96-well plates were centrifuged to pellet the splenocytes down and supernatant was transferred to a new 96 -well plate. The supernatant was stored at $-20^{\circ} \mathrm{C}$ until assayed. A Milliplex Mouse Th17 Luminex kit (MD MilliPore) with analytes IL$1 \beta$, IL-2, IL-4, IL-6, IL-10, IL-12(p70), IL-13, IL-17A, IL-23, IFN- $\gamma$, and TNF- $\alpha$ was used to quantify the cytokines secreted in the supernatant by the re-stimulated splenocytes. An adjusted protocol based on the manufacturers' recommendations was used with adjustments to use less sample and kit materials ${ }^{29}$. The readout was performed using a MagPix Luminex instrument. Raw data was analyzed using Bio-Plex Manager software, and further analysis was done with Excel and Prism.

\subsubsection{Cytokine production of activated CD4+ and CD8+T cells}

Surface staining and intracellular cytokine staining followed by flow cytometry were performed to measure the amount of activated (CD44+) CD4+ and CD8+ T cells producing IFN- $\gamma$, IL-2, TNF- $\alpha$, and IL-4 upon re-stimulation with S2RBD219 WT.

Five hours before the 24-hour re-stimulation incubation, Brefeldin A was added to block cytokines from secretion. PMA/I was also added to designated wells as a positive control. After the incubation, splenocytes were stained for the relevant markers. 
A viability dye and an Fc Block were also used to remove dead cells in the analysis and to minimize non-specific staining, respectively.

After staining, splenocytes were analyzed using an Attune NxT flow cytometer instrument at the Baylor College of Medicine Cytometry and Cell Sorting Core. Raw data was analyzed in VenturiOne software and gating results were copied in Excel. The $\%$-gating values from the non-stimulated controls were subtracted from the restimulated controls to observe the difference in \%-gating induced by the re-stimulation. The gating strategy from the analysis of the results is shown in Supplemental

Figure 1. From all events collected the doublets are removed to obtain only single-cell events. Then events are selected on size and granularity to obtain splenocytes only. Following the removal of dead splenocytes, a gate is set to only select activated $(\mathrm{CD} 44+) \mathrm{T}$ cells $(\mathrm{CD} 3+)^{30}$. T cells are then separated into CD4+ T helper cells and CD8+ cytotoxic T cells. For T helper cells the events positive for IFN- $\gamma$, TNF- $\alpha$, IL-2, and IL-4 were selected, while for cytotoxic T cells only IFN- $\gamma$, TNF- $\alpha$, and IL-2 positive events were gated.

\subsection{Statistical analysis}

To test for significant differences between groups in ELISA, Luminex, and flow cytometry results, Kruskal-Wallis tests in combination with Dunn's multiple comparison tests were performed. ns (not significant): $\mathrm{p}>0.05, *$ : $<0.05$ and $* *$ : $<<$ 0.01 . 


\section{Results}

Here we report on the expression of a modified, recombinant RBD of the SARS-CoV-2 spike protein using a yeast ( $P$. pastoris) expression system. The candidate antigen selection, modifications, and production processes were based on eight years of process development, manufacture, and preclinical prior experience with a SARS-CoV recombinant protein-based receptor-binding domain $(\mathrm{RBD})^{11-13}$. The RBDs of SARSCoV-2 and SARS $\square$ CoV share significant amino acid sequence similarity (>75\% identity, >80\% homology) and both use the human angiotensin-converting enzyme 2 (ACE2) receptor for cell entry ${ }^{31,32}$. Process development using the same procedures and strategies used for the production, scale-up, and manufacture of the SARS-CoV recombinant protein allowed for a rapid acceleration in the development of a scalable and reproducible production process for the SARS $\square$ CoV-2 RBD219-N1C1 protein, suitable for technological transfer to a manufacturer.

We found that the modifications used to minimize yeast-derived hyperglycosylation and optimize yield, purity, and stability of the SARS-CoV RBD219N1 protein were also relevant to the SARS-CoV-2 RBD expression and production process. The modified SARS-CoV-2 antigen, RBD219-N1C1, when formulated on Alhydrogel $^{\circledR}$, was shown to induce virus-neutralizing antibodies in mice, equivalent to those levels elicited by the wild-type (RBD219-WT) recombinant protein counterpart.

\subsection{Cloning and expression of the modified SARS-CoV-2 RBD}

The wild-type SARS-CoV-2 RBD amino acid sequence comprises residues 331-549 of the spike (S) protein (GenBank: QHD43416.1) of the Wuhan-Hu-1 isolate (GenBank: MN908947.3) ( Legends:

Figure 1). In the RBD-219-WT construct, the gene fragment was expressed in $P$. 
pastoris. After fermentation at the $5 \mathrm{~L}$ scale, the hexahistidine-tagged protein was purified by immobilized metal affinity chromatography, followed by size-exclusion chromatography. We observed glycosylation and aggregation during these initial expression and purification studies, and therefore, similar to our previous strategy ${ }^{12}$, we generated a modified construct, RBD219-N1C1, by deleting the N331 residue and mutating the $\mathrm{C} 538$ residue to alanine. The additional mutation of C538 to A538 was done because we observed that in the wild-type sequence nine cysteine residues likely would form four disulfide bonds. Therefore, the C538 residue was likely available for intermolecular cross-linking, leading to aggregation. As a result, in the RBD219-N1C1 construct, Pichia-derived hyperglycosylation, as well as aggregation via intermolecular disulfide bridging, were greatly reduced ${ }^{21}$. We note that the deleted and mutated residues are structurally far from the immunogenic epitopes and specifically the receptor-binding motif (RBM) of the RBD ( Legends:

Figure 1). On SDS-PAGE tris-glycine gels, the RBD219-WT protein migrated at approximately $28 \mathrm{kDa}$ under non-reduced conditions and $33 \mathrm{kDa}$ under reduced condition, while the RBD219-N1C1 protein migrated at approximately $24 \mathrm{kDa}$ under non-reduced condition and $29 \mathrm{kDa}$ under reduced condition. However, after N-glycans were removed enzymatically, these two proteins showed a similar molecular weight of approximately $25 \mathrm{kDa}$ (Supplemental Figure 2). The purity of both proteins was analyzed by densitometry showing levels of $>95 \%$.

\subsection{ACE-2 binds to recombinant $S A R S \square C o V-2$ RBD219-N1C1 protein} formulated on Alhydrogel ${ }^{\circledR}$

When mixing $25 \mu \mathrm{g}$ of either RBD219-WT or RBD219-N1C1 proteins with $500 \mu \mathrm{g}$ of 
Alhydrogel $^{\circledR}$, we observed that $>98 \%$ of the proteins bound to Alhydrogel ${ }^{\circledR}$ after 15 min of incubation. Only when the Alhydrogel ${ }^{\circledR}$ was reduced to less than $100 \mu \mathrm{g}$ (Alhydrogel ${ }^{\circledR} / \mathrm{RBD} 219$ ratio $\left.<4\right)$, the Alhydrogel ${ }^{\circledR}$ surface was saturated, and protein started to be detected in the supernatant (Figure 2A). It is known that unbound protein may impact the immunogenicity of the vaccine formulation, therefore we proceeded to only evaluate formulations with Alhydrogel ${ }^{\circledR} / \mathrm{RBD}_{219}$ ratios higher than 4.

Figure 2B shows that hACE-2-Fc, a recombinant version of the human receptor used by the virus to enter the host cells, can bind with the RBD proteins that are adsorbed on the surface of the Alhydrogel ${ }^{\circledR}$. This demonstrates that bound RBD proteins are structurally and possibly functionally active and that after adsorption the protein does not undergo any significant conformational changes that could result in the loss of possible key epitopes around the receptor-binding motif (RBM). The finding was consistent with the ACE-2 binding assay performed on the RBD proteins in the ELISA plate without being pre-adsorbed to Alhydrogel ${ }^{\circledR 21}$.

We saw no statistical differences between the binding of hACE-2-Fc to RBD219-WT (red, Figure 2B) or RBD219-N1C1 (green, Figure 2B) proteins, based on an unpaired t-test $(\mathrm{P}=0.670)$. Likewise, we saw no relation between the amount of Alhydrogel $^{\circledR}$ to which the RBD was bound and the interaction with hACE-2-Fc, indicating that the surface density of the RBD proteins on the Alhydrogel ${ }^{\circledR}$ plays no role in the presentation of ACE binding sites.

\subsection{Recombinant RBD219-N1C1 protein, formulated with Alhydrogel ${ }^{\circledR}$, elicits a strong neutralizing antibody response in mice}

Recombinant RBD219-N1C1 protein $(25 \mu \mathrm{g})$ was formulated with various amounts $(100-500 \mu \mathrm{g})$ of Alhydrogel ${ }^{\circledR}$. Controls included a cohort receiving only Alhydrogel ${ }^{\circledR}$ and another receiving the RBD219-WT antigen, also formulated with $500 \mu \mathrm{g}$ 
Alhydrogel $^{\circledR}$. Six- to eight-week-old female BALB/c mice were immunized 2-3 times subcutaneously at approximately 21-day intervals (Figure 3A). Blood samples were taken on day 35 from all study animals to assess total IgG antibody titers, as well as neutralizing antibody titers (Dataset 1). Half of the mice, those with the highest IgG titers in their respective group, were euthanized on day 43 to allow the evaluation of the cellular immune response after two immunizations. For this dataset (Dataset 2), we also measured IgG and neutralizing antibody titers. The remaining mice received a third vaccination on day 43 and were euthanized on day 57 for the assessment of humoral and cellular immune responses (Dataset 3).

Humoral immune response: On day 35 (Dataset 1), after receiving two vaccinations, all groups that had received the recombinant protein formulated with at least $200 \mu \mathrm{g}$ Alhydrogel ${ }^{\circledR}$ produced similar and robust IgG titers. The group receiving the protein with only $100 \mu \mathrm{g}$ Alhydrogel ${ }^{\circledR}$, produced a lower IgG response, albeit slightly higher than the negative control that had been immunized with $500 \mu \mathrm{g}$ Alhydrogel $^{\circledR}$ alone (Figure 3B, Supplemental Table 1). Importantly, based on a MannWhitney test, we determined that there was no statistical difference between the groups vaccinated with the modified and the wild-type version of the RBD protein $(\mathrm{p}=0.3497)$. The average neutralizing antibody titers observed on day 35 (IC50 range: 5.0x10 ${ }^{3}$ to 9.4x $10^{3}$, Supplemental Table 2) matched with the total IgG titers, showing equally high IC50 values for all vaccines that contained at least $200 \mu \mathrm{g}$ Alhydrogel ${ }^{\circledR}$ and lower IC50 values for the vaccine with only $100 \mu \mathrm{g}$ Alhydrogel ${ }^{\circledR}$ and no IC50 values for the adjuvant-only control (Figure 3C).

On day 43, 22 days after receiving the boost vaccination, half of the mice in each group $(\mathrm{N}=4)$, those with the highest IgG titers, were euthanized to determine the total $\mathrm{IgG}$, the IgG subtypes, and the neutralizing antibody titers. As we observed on day 
35, all animals that had received the vaccine produced strong antibody titers, with the groups receiving $\geq 200 \mu \mathrm{g}$ Alhydrogel ${ }^{\circledR}$ eliciting a higher titer than those that received only $100 \mu \mathrm{g}$ of Alhydrogel ${ }^{\circledR}$, albeit no statistical significance was detected (Figures 3B). For all animals, as typical for vaccine formulations containing aluminum, the IgG2a:IgG1 titer ratio was $<0.1$ (Supplemental Figure 3). In the pseudovirus neutralization assay for the day 43 samples (Figure 3C), all vaccines containing $\geq 200$ $\mu \mathrm{g}$ Alhydrogel ${ }^{\circledR}$ elicited IC50 titers that, on average, were several-fold higher than on day 35 (IC50 range: $1.1 \times 10^{4}$ to $1.2 \times 10^{5}$, Supplemental Table 2). There again was no difference between the RBD219-WT and RBD-N1C1 vaccines.

On day 57, all remaining animals were euthanized. In contrast to the animals studied on days 35 and 43, these animals had received a second boost vaccination. A robust immune response in all vaccinated mice, including those immunized with the protein adsorbed to $100 \mu \mathrm{g}$ Alhydrogel ${ }^{\circledR}$ achieved high average IgG titers. The total $\operatorname{IgG}$ titers in the mice euthanized on day 57 , had increased after the third vaccination, compared to the titers seen on day 35. Likewise, we observed a corresponding increase in the average IC50 values (IC50 range: $3.8 \times 10^{2}$ to $1.1 \times 10^{4}$, Supplemental Table 2 ) for all animals, including those immunized with the protein adsorbed to $100 \mu \mathrm{g}$ Alhydrogel $^{\circledR}$. Interestingly, for this time point, the cohort receiving $25 \mu \mathrm{g}$ RBD219N1C1 with $500 \mu \mathrm{g}$ Alhydrogel ${ }^{\circledR}$ appeared to show higher neutralizing antibody titers than the corresponding RBD219-WT group, albeit that difference was not statistically significant.

Cellular immune response: For all animals euthanized on day 43 (having received two vaccinations) and day 57 (having received three vaccinations), the cellular immune response was characterized through the restimulation of isolated mouse splenocytes with the recombinant RBD219-WT protein. For all samples, we employed 
Flow Cytometry to quantify intracellular cytokines in $\mathrm{CD} 4+$ and $\mathrm{CD} 8^{+}$cells after restimulation (Figure 4A). On day 43, high percentages of CD4 ${ }^{+}$-IL-4 and, to a slightly lesser extent $\mathrm{CD}^{+}{ }^{+} \mathrm{TNF} \alpha$ producing cells were detected. Conversely, as expected for an Alhydrogel ${ }^{\circledR}$-adjuvanted vaccine, low levels of IL-2 producing CD4 ${ }^{+}$cells were seen. In a cytokine release assay, strong IFN- $\gamma$, IL-6, and IL-10 secretion was observed independent of whether the animals had received two or three immunizations, whereas low amounts of secreted Th1-typical cytokines such as IL-2 or IL-12 were seen (Figure 4B).

\subsection{Study limitations}

The authors recognize that the data presented here has limitations. For instance, we do not include viral challenge studies. Prediction of the vaccine's in vivo efficacy is therefore based on the neutralizing activity of the vaccine-induced antibodies in mice, measured through an in vitro pseudovirus assay. A challenge study in non-human primates is currently ongoing and will be published separately. Moreover, our studies were limited to aluminum-based formulations. Further studies are needed to investigate the combination of the vaccine antigen with different adjuvant and immunostimulant formulations, which may lead to dose spearing, reduce the number of doses, and/or induce a more balanced and long-lasting immune response. 


\section{Discussion}

Here we report on a yeast-expressed SARS-CoV-2 RBD219-N1C1 protein and its potential as a vaccine antigen candidate for preventing COVID-19. Building on extensive prior experience developing vaccines against SARS-CoV and MERS-CoV ${ }^{12-}$ ${ }^{14}$, we selected and compared the SARS-CoV-2 RBD219-WT and the SARS-CoV-2 RBD219-N1C1 proteins for their potential to induce high titers of virus-neutralizing antibodies, T-cell responses, and protective immunity. The decision to focus our antigen development on the RBD of the viral spike protein stems in part from earlier work on the ancestral SARS vaccine, where we had already been able to demonstrate low-cost, high-yield production of that homologous vaccine antigen ${ }^{33}$, something that we now also confirmed formally for the SARS-CoV-2 RBD antigen ${ }^{34}$. In addition, the original SARS-CoV RBD-based vaccine was superior to the full-length spike protein at inducing specific antibodies and fully protected mice from SARS-CoV infection while preventing eosinophilic pulmonary infiltrates in the lungs upon challenge ${ }^{11}$, a process more likely linked to Th17-dominant and mixed Th1/Th2 responses, when compared to using full-length spike protein as the antigen ${ }^{35}$. As an additional argument in favor of the RBD, Liu et al. have reported that most neutralizing epitopes of the SARS-CoV-2 spike protein are in the RBD and the N-terminal domain (NTD), with the most potent ones near the RBD's Receptor Binding Motif ${ }^{36}$.

While it is known that yeast $\mathrm{N}$-glycosylation is different from that in mammalian cells and yeast-derived glycosylation of the eight $\mathrm{N}$-glycosylation sites in the NTD might alter the induction of neutralizing antibodies, little impact is expected for the RBD since there are no glycosylation sites near the RBM. Moreover, in this work, using the SARS-CoV-2 RBD219 protein homolog, we observed that, just like in the case of the SARS-CoV RBD antigen, the deletion of the N-terminal asparagine residue reduced hyperglycosylation, thus allowing for easier purification of the antigen 
obtained from the yeast expression system. This finding was confirmed when comparing the minimal changes noted in apparent molecular weights of our reduced RBD compared to an enzymatically deglycosylated form. As an additional modification, mutagenesis of a free cysteine residue further improved protein production through the reduction of aggregation. Based on the predicted structure of the RBD, no impact on the functionality of the RBD219-N1C1 antigen was expected, and using an ACE-2 in vitro binding assay we indeed showed similarity to the RBD219-WT antigen. In addition, we showed that, in mice, the modified RBD219-N1C1 antigen triggered an equivalent immune response to the RBD219-WT protein when both proteins were adjuvanted with Alhydrogel ${ }^{\circledR}$.

Similar to our previous findings with the SARS-CoV RBD antigen ${ }^{11}$, we show that RBD219-N1C1 when formulated with Alhydrogel ${ }^{\circledR}$ elicits a robust neutralizing antibody response with IC50 values up to $4.3 \times 10^{5}$ in mice, as well as an expected T-cell immunological profile. Some of the titers of virus-neutralizing antibodies exceed the titer, $2.4 \times 10^{4}$, measured in-house with human convalescent serum research reagent for SARS-CoV-2 (NIBSC 20/130, National Institute for Biological Standards and Control, UK). Among the T-cell activation markers, we found high percentages of CD4+ T cells expressing IL-4 and IFN- $\gamma$, possibly indicating increased numbers of functional T follicular helper cells which will support the generation of antibody-producing plasma cells and longlived memory B cells ${ }^{37}$.

In a mouse virus challenge model for the SARS-CoV RBD recombinant protein vaccine, we found that Alhydrogel ${ }^{\circledR}$ formulations induced high levels of protective immunity but did not stimulate eosinophilic immune enhancement, suggesting that Alhydrogel ${ }^{\circledR}$ may even reduce immune enhancement. This prior experience offers the potential for Alhydrogel ${ }^{\circledR}$ as a key adjuvant for consideration during coronavirus vaccine development ${ }^{38}$. Such findings have led to a reframing of the basis for immune 
enhancement linked to coronavirus respiratory infections ${ }^{35}$. A recent analysis and review by the NIH ACTIV Vaccine Working Group confirmed that aluminum or Th2 responses remain viable options for vaccine development concluding that "it is not possible to clearly prioritize or down-select vaccine antigens, adjuvants, biotechnology platforms, or delivery mechanisms based on general immunological principles or the available preclinical data"39.

Therefore, the RBD219-N1C1 vaccine antigen on Alhydrogel ${ }^{\circledR}$ merits its evaluation as a COVID-19 vaccine with or without other immunostimulants. Looking at the landscape of recombinant protein-based COVID-19 vaccines, the WHO lists several advanced COVID-19 candidates that are based on recombinant proteins ${ }^{1}$, and at least seven COVID-19 vaccines include aluminum as part of the adjuvant component ${ }^{8,15,17}$, 18, 40-46, often in combination with other immunostimulants, such as $\mathrm{CpG}$, to achieve a balanced immune response. These recombinant protein vaccines, including RBD219$\mathrm{N} 1 \mathrm{C} 1$, might find an additional important use as a booster if one of the newer platform vaccines, e.g., mRNA or adenovirus-based vaccines induce lower than expected immunogenicity or protection. Likewise, we see an opportunity for protein vaccines representing the newly appearing SARS-CoV-2 variants ${ }^{47}$ to act as boosters in individuals previously immunized with the wild-type antigen. Such prime-boost approaches have been used successfully with the chimp adenovirus vaccine for malaria and other systems ${ }^{48,49}$, and are currently being entertained for SARS-CoV- $2^{50}$.

The selection of the P. pastoris expression platform for the production of the RBD antigen was motivated by the intent to develop a low-cost production process that could easily be transferred to manufacturers in LMICs. Currently, there are several types of COVID-19 vaccine candidates in advanced clinical trials ${ }^{7,51-56}$ that, as they require advanced infrastructure, focus on the developed world. Being able to match the 
existing experience in LMICs with the production of other biologics in yeast increases the probability of successful technology transfer. For example, currently, the recombinant hepatitis $\mathrm{B}$ vaccine is produced in yeast by several members of the Development Country Vaccine Manufacturers Network (DCVMN), and we foresee that, given the existing infrastructure and expertise, those facilities could be repurposed to produce a yeast-produced COVID-19 vaccine ${ }^{58}$. Recently, the research cell bank and the production process for the RBD219-N1C1 antigen were technologically transferred to a vaccine manufacturer in India and produced under cGMP conditions; adjuvanted with Alum and CpG and that vaccine is currently in clinical trials in India ${ }^{59}$. In parallel, studies to adapt the RBD219-WT and RBD219-N1C1 antigens to the recently appearing SARS-CoV-2 variants are underway, as is a SARS-CoV-2 challenge study in a nonhuman primate model. Given the limited access to suitable test facilities, the onset of that study was significantly delayed, but we expect to publish its results soon. 


\section{Acknowledgments:}

This work was supported by the Robert J. Kleberg Jr. and Helen C. Kleberg Foundation, as well as the NIH (AI14087201), Baylor College of Medicine, and Texas Children's Hospital. This project was further supported by the Cytometry and Cell Sorting Core at Baylor College of Medicine with funding from the CPRIT Core Facility Support Award (CPRIT-RP180672), the NIH (CA125123 and RR024574), and the assistance of Joel M. Sederstrom. We are grateful to Dr. Vincent Munster (NIAID) for providing the spike expression plasmid for SARS-CoV-2.

\section{Declaration of interest statement:}

The authors declare they are developers of the RBD219-N1C1 technology, and that Baylor College of Medicine recently licensed it to Biological E, an Indian manufacturer for further advancement and licensure. The research conducted in this paper was performed in the absence of any commercial or financial relationships that could be construed as a potential conflict of interest.

\section{References:}

1. WHO. The COVID-19 candidate vaccine landscape. 2020.

2. Heaton PM. The Covid-19 Vaccine-Development Multiverse. The New England journal of medicine 2020 .

3. Chen WH, Strych U, Hotez PJ, Bottazzi ME. The SARS-CoV-2 Vaccine Pipeline: an Overview. Current tropical medicine reports 2020:1-4.

4. Slaoui M, Hepburn M. Developing Safe and Effective Covid Vaccines Operation Warp Speed's Strategy and Approach. The New England journal of medicine 2020.

5. WHO. ACCESS TO COVID-19 TOOLS (ACT) ACCELERATOR 2020.

6. Gao Q, Bao L, Mao H, Wang L, Xu K, Yang M, et al. Development of an inactivated vaccine candidate for SARS-CoV-2. Science 2020; 369:77-81.

7. Keech C, Albert G, Cho I, Robertson A, Reed P, Neal S, et al. Phase 1-2 Trial of a SARS-CoV-2 Recombinant Spike Protein Nanoparticle Vaccine. The New England journal of medicine 2020 .

8. Yang J, Wang W, Chen Z, Lu S, Yang F, Bi Z, et al. A vaccine targeting the RBD of the S protein of SARS-CoV-2 induces protective immunity. Nature 2020. 
9. Li T, Zheng Q, Yu H, Wu D, Xue W, Xiong H, et al. SARS-CoV-2 spike produced in insect cells elicits high neutralization titres in non-human primates. Emerging microbes \& infections 2020; 9:2076-90.

10. Pollet J, Chen WH, Strych U. Recombinant protein vaccines, a proven approach against coronavirus pandemics. Adv Drug Deliv Rev 2021; 170:71-82.

11. Chen WH, Tao X, Agrawal A, Algaissi A, Peng BH, Pollet J, et al. YeastExpressed SARS-CoV Recombinant Receptor-Binding Domain (RBD219-N1) Formulated with Alum Induces Protective Immunity and Reduces Immune Enhancement. Vaccine 2020; 22:31232-9.

12. Chen WH, Du L, Chag SM, Ma C, Tricoche N, Tao X, et al. Yeast-expressed recombinant protein of the receptor-binding domain in SARS-CoV spike protein with deglycosylated forms as a SARS vaccine candidate. Human vaccines \& immunotherapeutics 2014; 10:648-58.

13. Chen WH, Chag SM, Poongavanam MV, Biter AB, Ewere EA, Rezende W, et al. Optimization of the Production Process and Characterization of the Yeast-Expressed SARS-CoV Recombinant Receptor-Binding Domain (RBD219-N1), a SARS Vaccine Candidate. Journal of pharmaceutical sciences 2017.

14. Nyon MP, Du L, Tseng CK, Seid CA, Pollet J, Naceanceno KS, et al. Engineering a stable $\mathrm{CHO}$ cell line for the expression of a MERS-coronavirus vaccine antigen. Vaccine 2018; 36:1853-62.

15. CHICTR. Randomized double blind, placebo controlled phase I trial for anti novel coronavirus pneumonia (COVID-19) recombinant vaccine (Sf9). 2020.

16. Clinicaltrials_gov. KBP-201 COVID-19 Vaccine Trial in Healthy Volunteers. 2021.

17. Clinicaltrials_gov. A Study to Evaluate the Safety and Immunogenicity of COVID-19 (AdimrSC-2f) Vaccine. 2021.

18. Clinicaltrials_gov. Clinical Study of Recombinant Novel Coronavirus Vaccine. 2020.

19. RPCEC. Soberano 01 - Estudio Fase I/II, aleatorizado, controlado, adaptativo, a doble ciego y multicéntrico para evaluar la seguridad, reactogenicidad e inmunogenicidad del Candidato Vacunal profiláctico FINLAY- FR-1 anti SARS - CoV - 2 en un esquema de dos dosis. (COVID-19). 2020.

20. He Y, Zhou Y, Liu S, Kou Z, Li W, Farzan M, et al. Receptor-binding domain of SARS-CoV spike protein induces highly potent neutralizing antibodies: implication for developing subunit vaccine. Biochem Biophys Res Commun 2004; 324:773-81.

21. Chen WH, Wei J, Kundu R, Adhikari R, Liu Z, Lee J, et al. Cloning, Expression and Biophysical Characterization of a Yeast-expressed Recombinant SARS-CoV-2 Receptor Binding Domain COVID-19 Vaccine Candidate. bioRxiv 2020; https://www.biorxiv.org/content/10.1101/2020.11.09.373449v1.

22. Hotez PJ, Bottazzi ME. Developing a low-cost and accessible COVID-19 vaccine for global health. PLoS neglected tropical diseases 2020; 14:e0008548.

23. Hotez PJ, Bottazzi ME, Singh SK, Brindley PJ, Kamhawi S. Will COVID-19 become the next neglected tropical disease? PLoS neglected tropical diseases 2020; 14:e0008271. 
24. Millet JK, Whittaker GR. Murine Leukemia Virus (MLV)-based Coronavirus Spike-pseudotyped Particle Production and Infection. Bio-protocol 2016; 6.

25. Letko M, Marzi A, Munster V. Functional assessment of cell entry and receptor usage for SARS-CoV-2 and other lineage B betacoronaviruses. Nat Microbiol 2020; 5:562-9.

26. He J, Choe S, Walker R, Di Marzio P, Morgan DO, Landau NR. Human immunodeficiency virus type 1 viral protein $\mathrm{R}(\mathrm{Vpr})$ arrests cells in the $\mathrm{G} 2$ phase of the cell cycle by inhibiting p34cdc2 activity. J Virol 1995; 69:6705-11.

27. Zufferey R, Nagy D, Mandel RJ, Naldini L, Trono D. Multiply attenuated lentiviral vector achieves efficient gene delivery in vivo. Nature biotechnology 1997; 15:871-5.

28. Jones K, Versteeg L, Damania A, Keegan B, Kendricks A, Pollet J, et al. Vaccine-Linked Chemotherapy Improves Benznidazole Efficacy for Acute Chagas Disease. Infection and immunity 2018; 86.

29. Versteeg L, Le Guezennec X, Zhan B, Liu Z, Angagaw M, Woodhouse JD, et al. Transferring Luminex(R) cytokine assays to a wall-less plate technology: Validation and comparison study with plasma and cell culture supernatants. J Immunol Methods 2017; 440:74-82.

30. Schumann J, Stanko K, Schliesser U, Appelt C, Sawitzki B. Differences in CD44 Surface Expression Levels and Function Discriminates IL-17 and IFN-gamma Producing Helper T Cells. PLoS One 2015; 10:e0132479.

31. Zhou P, Yang XL, Wang XG, Hu B, Zhang L, Zhang W, et al. A pneumonia outbreak associated with a new coronavirus of probable bat origin. Nature 2020; 579:270-3.

32. Hoffmann M, Kleine-Weber H, Schroeder S, Kruger N, Herrler T, Erichsen S, et al. SARS-CoV-2 Cell Entry Depends on ACE2 and TMPRSS2 and Is Blocked by a Clinically Proven Protease Inhibitor. Cell 2020; 181:271-80 e8.

33. Chen WH, Chag SM, Poongavanam MV, Biter AB, Ewere EA, Rezende W, et al. Optimization of the Production Process and Characterization of the Yeast-Expressed SARS-CoV Recombinant Receptor-Binding Domain (RBD219-N1), a SARS Vaccine Candidate. Journal of pharmaceutical sciences 2017; 106:1961-70.

34. Lee J, Liu Z, Chen WH, Wei J, Kundu R, Adhikari R, et al. Process Development and Scale-up Optimization of the SARS-CoV-2 Receptor Binding Domain-Based Vaccine Candidate, RBD219-N1C1. biorxiv 2021; https://www.biorxiv.org/content/10.1101/2020.12.30.424829v1.

35. Hotez PJ, Bottazzi ME, Corry DB. The potential role of Th17 immune responses in coronavirus immunopathology and vaccine-induced immune enhancement. Microbes and infection 2020.

36. Liu L, Wang P, Nair MS, Yu J, Rapp M, Wang Q, et al. Potent neutralizing antibodies against multiple epitopes on SARS-CoV-2 spike. Nature 2020; 584:450-6.

37. Karlsson AC, Humbert $\mathrm{M}$, Buggert $\mathrm{M}$. The known unknowns of $\mathrm{T}$ cell immunity to COVID-19. Sci Immunol 2020; 5.

38. Hotez PJ, Corry DB, Strych U, Bottazzi ME. COVID-19 vaccines: neutralizing antibodies and the alum advantage. Nature reviews Immunology 2020; 20:399-400. 
39. Haynes BF, Corey L, Fernandes P, Gilbert PB, Hotez PJ, Rao S, et al. Prospects for a safe COVID-19 vaccine. Sci Transl Med 2020.

40. Liang JG, Su D, Song TZ, Zeng Y, HUang W, Wu J, et al. S-Trimer, a COVID19 subunit vaccine candidate, induces protective immunity in nonhuman primates. bioRxiv 2020.

41. Clinicaltrials_gov. SCB-2019 as COVID-19 Vaccine. 2020.

42. Genetic_Engineering\&Biotechnology_News.COVAXX - UB-612. 2020.

43. Clinicaltrials_gov. A Study to Evaluate the Safety and Immunogenicity of MVC-COV1901 Against COVID-19. 2020.

44. Clinicaltrials_gov. Study of the Safety, Reactogenicity and Immunogenicity of "EpiVacCorona" Vaccine for the Prevention of COVID-19 (EpiVacCorona). 2020.

45. Clinicaltrials_gov. A Study to Evaluate the Safety, Tolerability, and Immunogenicity of UB-612 COVID-19 Vaccine. 2020.

46. Kuo TY, Lin MY, Coffman RL, Campbell JD, Traquina P, Lin YJ, et al. Development of CpG-adjuvanted stable prefusion SARS-CoV-2 spike antigen as a subunit vaccine against COVID-19. Sci Rep 2020; 10:20085.

47. Garry RF. Mutations arising in SARS-CoV-2 spike on sustained human-tohuman transmission and human-to-animal passage. Virologicalorg, 2021.

48. Draper SJ, Biswas S, Spencer AJ, Remarque EJ, Capone S, Naddeo M, et al. Enhancing blood-stage malaria subunit vaccine immunogenicity in rhesus macaques by combining adenovirus, poxvirus, and protein-in-adjuvant vaccines. Journal of immunology 2010; 185:7583-95.

49. Hodgson SH, Choudhary P, Elias SC, Milne KH, Rampling TW, Biswas S, et al. Combining viral vectored and protein-in-adjuvant vaccines against the blood-stage malaria antigen AMA1: report on a phase 1a clinical trial. Molecular therapy : the journal of the American Society of Gene Therapy 2014; 22:2142-54.

50. Gov.UK. World-first COVID-19 alternating dose vaccine study launches in UK. 2021.

51. van Doremalen N, Lambe T, Spencer A, Belij-Rammerstorfer S, Purushotham JN, Port JR, et al. ChAdOx1 nCoV-19 vaccine prevents SARS-CoV-2 pneumonia in rhesus macaques. Nature 2020.

52. Sadoff J, De Paepe E, Haazen W, Omoruyi E, Bastian AR, Comeaux C, et al. Safety and Immunogenicity of the Ad26.RSV.preF Investigational Vaccine Coadministered With an Influenza Vaccine in Older Adults. The Journal of infectious diseases 2020.

53. Folegatti PM, Ewer KJ, Aley PK, Angus B, Becker S, Belij-Rammerstorfer S, et al. Safety and immunogenicity of the ChAdOx1 $\mathrm{nCoV}-19$ vaccine against SARS-CoV2: a preliminary report of a phase $1 / 2$, single-blind, randomised controlled trial. Lancet 2020; 396:467-78.

54. Jackson LA, Anderson EJ, Rouphael NG, Roberts PC, Makhene M, Coler RN, et al. An mRNA Vaccine against SARS-CoV-2 - Preliminary Report. The New England journal of medicine 2020 . 
55. Corbett KS, Edwards DK, Leist SR, Abiona OM, Boyoglu-Barnum S, Gillespie RA, et al. SARS-CoV-2 mRNA vaccine design enabled by prototype pathogen preparedness. Nature 2020; 586:567-71.

56. Walsh EE, Frenck RW, Jr., Falsey AR, Kitchin N, Absalon J, Gurtman A, et al. Safety and Immunogenicity of Two RNA-Based Covid-19 Vaccine Candidates. The New England journal of medicine 2020; 383:2439-50.

57. Hotez PJ, Bottazzi ME. Developing a Low-Cost and Accessible COVID-19 Vaccine for Global Health. Preprintsorg, 2020.

58. WHO. Prequalified Vaccines. 2020.

59. CTRI. Biological E's novel Covid-19 vaccine of SARS-CoV-2 for protection against Covid-19 disease., 2020. 


\section{Figure Legends:}

Figure 1 A) Amino acid sequence alignment between SARS-CoV-2 RBD219-WT (S2RBD) and RBD219-N1C1 (S2-RBD-N1C1). In the N1C1-mutant, the N-terminal glutamine residue (N331, green) is removed and a C538A mutation (yellow) was introduced. Neither mutation is inside the receptor-binding motif (RBM, purple). B) The structure model of RBD219-WT was extracted from the crystal structure of the SARS-CoV-2 spike protein (PDB ID 6VXX). The RBM (N436-Y508) is again shown in purple while the deleted asparagine (N331) and mutated cysteine (C538, mutated to alanine) in RBD219-N1C1 are highlighted in green and yellow, respectively.

Figure 2 A) Langmuir binding isotherm of RBD219-N1C1 to Alhydrogel ${ }^{\circledR}$. B) ELISA data, comparing the binding interaction of hACE-2-Fc to RBD219-WT bound Alhydrogel $^{\circledR}$ (red) and RBD219-N1C1 bound on different amounts of Alhydrogel ${ }^{\circledR}$ (green, purple, orange, and black). Five hundred $\mu \mathrm{g}$ Alhydroge ${ }^{\circledR}$ alone served as a negative control (blue). Data are shown as the geometric mean $(n=3)$ with $95 \%$ confidence intervals.

Figure 3. A) Study design. B) Total IgG titers of Datasets 1, 2, and 3 measured respectively at days 35,43 , and 57 post the prime injection. IgG titers were determined against RBD219-WT protein. Closed data points represent data from mice with the highest IgG titers (Dataset 2), open data points represent data from mice with the lowest IgG titers (Dataset 3). C) IC50 values measured by a pseudovirus neutralization assay. Datasets 1, 2, and 3 are measured respectively at day 35, 43, and 57 after the first injection. Baselines indicate the lowest dilution measured. Lines on each group represent the geometric mean and $95 \%$ confidence intervals.

Figure 4: A) Heatmap of the cytokine response of CD4+ and CD8+ T cells after restimulation with SARS-CoV-2 RBD219-WT or RBD219-N1C1, re-stimulated splenocytes were surface and intracellularly stained and subsequently analyzed by flow 
cytometry. Splenocytes were obtained from mice who received two vaccinations (day 43) or three vaccinations (Day 57). Non-stimulated controls were subtracted from restimulated samples. B) Heatmap of secreted cytokines in supernatant from re-stimulated splenocytes from mice who received two vaccinations (day 43) or three vaccinations (Day 57). Cytokine concentrations of non-stimulated controls were subtracted from restimulated samples. 
$\begin{array}{ll}\text { A) } & \text { B) }\end{array}$

S2-RBD S2-RBD-N1C1

S2-RBD S2-RBD-N1C1

S2-RBD $\mathrm{S} 2-\mathrm{RBD}-\mathrm{N} 1 \mathrm{C} 1$

S2-RBD S2-RBD-N1C1

S2-RBD S2-RBD-N1C1
331 NITNLCPFGE VFNATRFASV YAWNRKRISN CVADYSVLYN SASFSTFKCY 380 331 ITNLCPFGE VFNATRFASV YAWNRKRISN CVADYSVLYN SASFSTFKCY 380

381 GVSPTKLNDL CFTNVYADSF VIRGDEVRQI APGQTGKIAD YNYKLPDDFT 430 381 GVSPTKLNDL CFTNVYADSF VIRGDEVRQI APGQTGKIAD YNYKLPDDFT 430

431 GCVIAWNSNN LDSKVGGNYN YLYRLFRKSN LKPFERDIST EIYQAGSTPC 480 431 GCVIAWNSNN LDSKVGGNYN YLYRLFRKSN LKPFERDIST EIYQAGSTPC 480

481 NGVEGENCYF PLQSYGEQPT NGVGYQPYRV VVLSFELLHA PATVCGPKKS 530 481 NGVEGENCYE PLQSYGEQPT NGVGYQPYRV VVLSFELLHA PATVCGPKKS 530

531 TNLVKNKCVN FNFNGLTGT 549

531 TNLVKNKAVN FNFNGLTGT 549

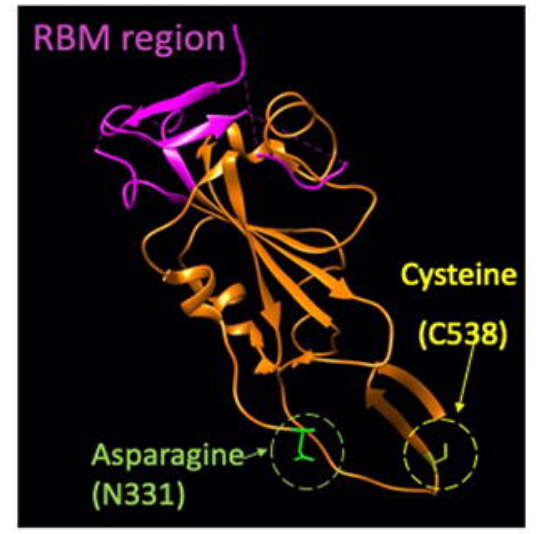




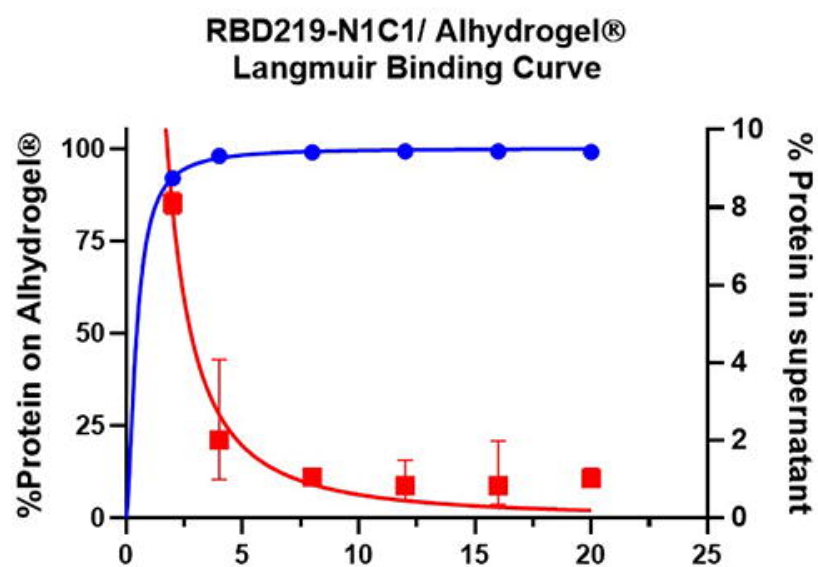

Alhydrogel/RBD219-N1C1 ratio $(\mathrm{mg} / \mathrm{mg})$

$\%$ Protein on Alhydrogel $(\AA$

$\%$ Protein in supernatant
Binding of hACE2-Fc to RBD/Alhydrogel ${ }^{(}$

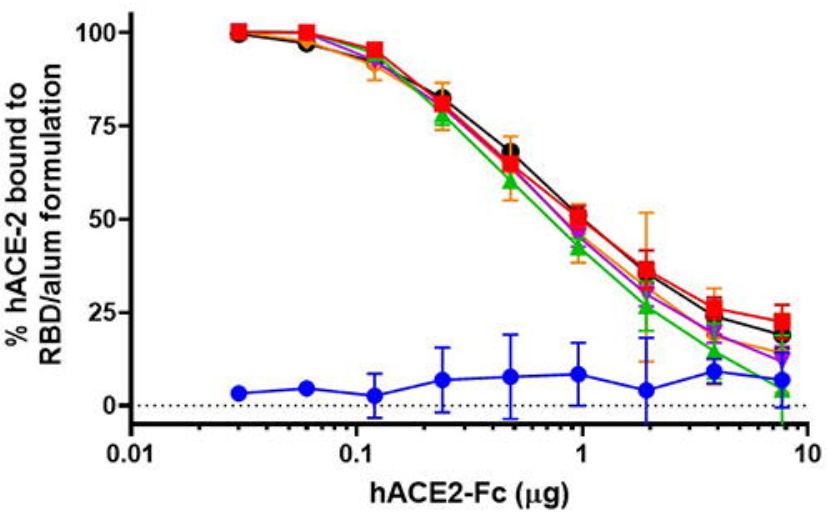

- $500 \mu \mathrm{g}$ Alhydrogel\&

$25 \mu \mathrm{g}$ RBD219-WT+His $+500 \mu \mathrm{g}$ Alhydrogel $\&$

- $25 \mu \mathrm{g}$ RBD219-N1C1 $+400 \mu \mathrm{g}$ Alhydrogel $\otimes$

$25 \mu \mathrm{g}$ RBD219-N1C1 $+200 \mu \mathrm{g}$ Alhydrogel $\otimes$

$25 \mu \mathrm{g}$ RBD219-N1C1 $+500 \mu \mathrm{g}$ Alhydrogel®
$25 \mu \mathrm{g}$ RBD219-N1C1

$+100 \mu \mathrm{g}$ Alhydrogel 8 


\section{A) Cytokines production by stimulated T-cells}

Dataset 2: mice with high IgG titers after $1^{\text {st }}$ boost (Day 35), (sacrificed on Day 43)

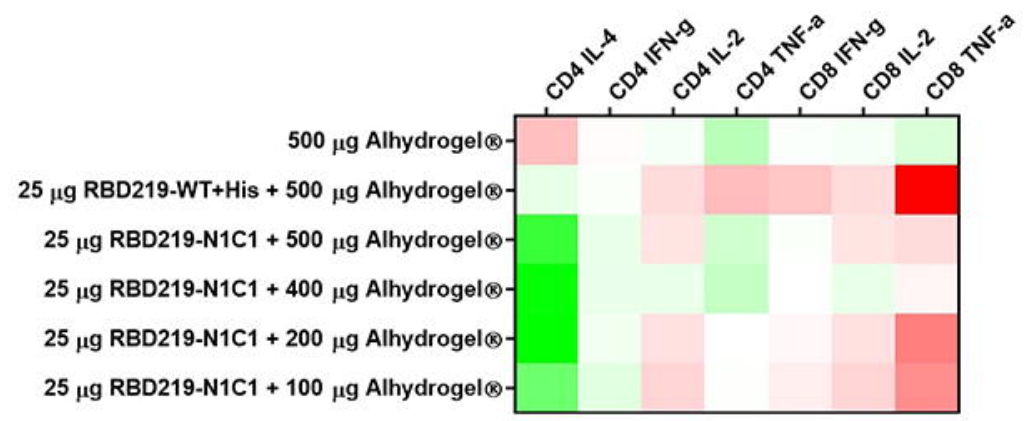

Dataset 3: mice with low IgG titers at 1st boost (Day 35), that received $2^{\text {nd }}$ boost on Day 43 (sacrificed on Day 57)

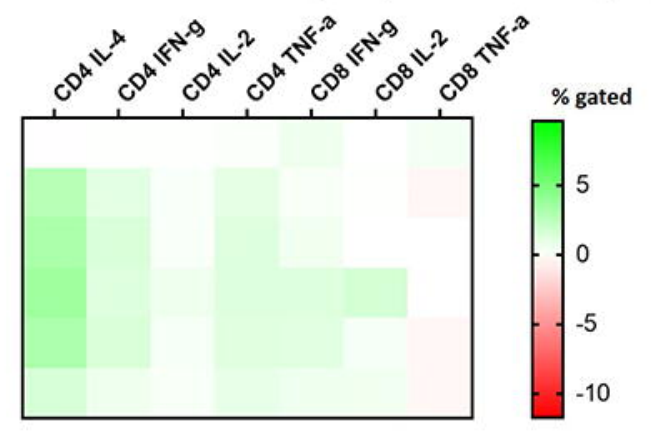

Dataset 3: mice with low IgG titers at 1st boost (Day 35), that received $2^{\text {nd }}$ boost on Day 43 (sacrificed on Day 57)

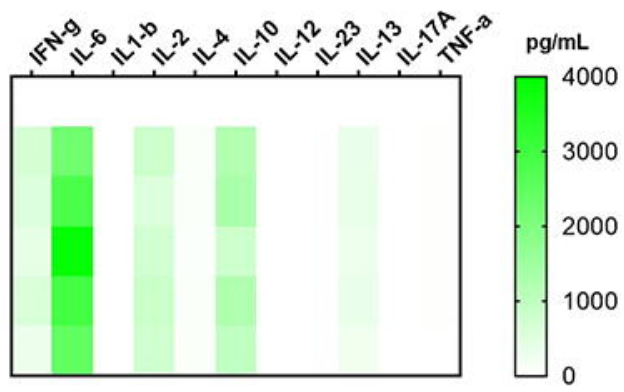

$$
\text { that received } 2^{\text {nd }} \text { boost on Day } 43 \text { (sacrificed on Day 57) }
$$

\section{B) Cytokines secreted by stimulated splenocytes}

Dataset 2: mice with high $\operatorname{lgG}$ titers after $1^{\text {st }}$ boost (Day 35 ), (sacrificed on Day 43)

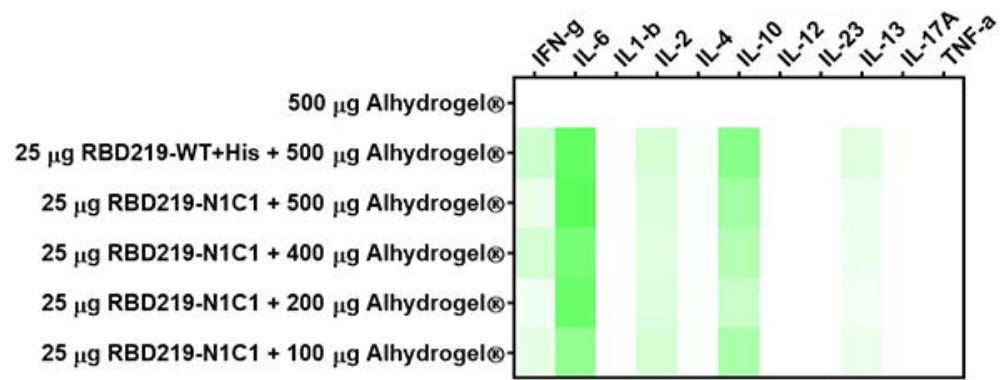

